\title{
Kinematic properties and stellar populations of dwarf and faint early-type galaxies in the Coma cluster
}

\author{
Ana Matković and Rafael Guzmán
}

\author{
Department of Astronomy, University of Florida, PO Box 112055, Gainesville, \\ FL 32611-2055, USA \\ email: matkovic@astro.ufl.edu and guzman@astro.ufl.edu
}

\begin{abstract}
We obtained velocity dispersion and line strength index measurements for 69 faint early-type galaxies in the core of the Coma cluster. The full sample spanning $-22.0<M_{R}<$ $-17.5 \mathrm{mag}$ includes $36 \mathrm{dE} / \mathrm{dS} 0$ galaxies. We examine the $L-\sigma$ relation and compare it to that of bright ellipticals from the literature. The slope defined by the faint early-type galaxies including dEs/dS0s is significantly shallower, $L \propto \sigma^{2.01 \pm 0.36}$, than that defined by luminous elliptical galaxies, $L \propto \sigma^{4}$. Moreover, there is no evidence for a change of slope at the typical division between dwarfs and ordinary elliptical galaxies at $M_{B}=-18$ mag; rather, the change in slope appears at $M_{B}=-20.5 \mathrm{mag}$. We show that rotation in the fainter galaxies cannot be solely responsible for the change of slope in the $L-\sigma$ relation. We also plot metallicity and age-dependent line strength indices in diagnostic diagrams and find that our galaxies span a wide range in both age and metallicity.
\end{abstract}

Keywords. kinematics, clusters: individual (Coma), dwarf, elliptical and lenticular, formation, fundamental parameters.

\section{Introduction}

The kinematic properties of dwarf elliptical galaxies (dEs) offer crucial insight into galaxy formation scenarios. Unfortunately, their low surface brightness makes observations, particularly those useful to kinematic studies, challenging. Despite this difficulty, several studies measuring the velocity dispersions of dEs have recently emerged, the latest two being De Rijcke et al. (2004) and Smith et al. (2004). To further this research, we present a statistically representative sample of faint early-type galaxies in different environments within one cluster, an essential tool for testing different formation mechanisms of $\mathrm{dE}$ galaxies.

The primary goal of this study is to characterize kinematic and stellar populations of dwarf and other low-luminosity early-type galaxies as a function of their environment. For this purpose we observed galaxies in the center and outskirts of the Coma cluster. Here we present our preliminary results, which concentrate on the central region.

\section{Observations and Data Reduction}

We observed the Coma cluster faint early-type galaxies with the HYDRA multi-fiber spectrograph on the $3.5 \mathrm{~m}$ WIYN telescope at Kitt Peak National Observatory. We obtained galaxies in two 45 arcmin diameter regions in the center and outskirts of the Coma cluster. The selection of the faint early-type galaxies was done using the $C-M$, and $C-C$ diagrams containing the $\mathrm{U}, \mathrm{B}$, and R bands (see Matković \& Guzmán 2005 for more 
detail). In our sample, we define faint early-type galaxies to have $-15.8 \geqslant M_{B} \geqslant-20.5$ mag $\dagger$ where 36 out of 69 of these objects are dwarf galaxies $\left(M_{B}>-18\right.$, Ferguson \& Binggeli 1994). We define the galaxies brighter than $M_{B} \leqslant-20.5$ as bright ellipticals.

The wavelength range of $4120-5600 \AA$ was optimal for discerning a few of the most prominent features of early-type galaxies: G-band, $\mathrm{H} \beta, \mathrm{Mg}_{2}$, and $\mathrm{Fe} \lambda 5350$. The FWHM of $1.91 \AA$ allowed us to detect velocity dispersions down to $35 \mathrm{~km} \mathrm{~s}^{-1}$. Due to the low throughput of the fibers and to reach $S / N>15$, our galaxies had long exposure times, ranging from 4 to 18 hours.

We reduced all the spectra using the 'dohydra' task within IRAF, while we measured the velocity dispersions $(\sigma)$ with the REDUCEME software (Cardiel 1999). The software implements the Fourier Quotient method. We determined the uncertainty in the $\sigma$ measurements using the boot-strapping technique.

\section{3. $L-\sigma$ Relation}

Faber \& Jackson (1976) showed that the bright early-type galaxies exhibit a relation between their luminosity and velocity dispersion such that $L \propto \sigma^{n}$, where $n$ was thought to be $\approx 4$ (Faber \& Jackson 1976; Sargent et al. 1977; Schechter \& Gunn 1979; Schechter 1980; Tonry \& Davis 1981; Terlevich et al. 1981). In 1981, Tonry (1981) observed that the faint early-type galaxies followed a slightly shallower relation than the giant ellipticals, $L \propto \sigma^{3}$. This result was confirmed by Davies et al. (1983) and Held et al. (1992) whose samples yielded $n \approx 2.5$. Both Tonry (1981) and Davies et al. (1983) discussed rotation as a possible cause for the change of slope in the $L-\sigma$ relation, while Held et al. (1992) found it consistent with the supernova driven galactic winds models. The data samples of these authors only included approximately a dozen dEs.

Here we show the $L-\sigma$ relation for our sample of 69 faint early-type galaxies in the central 45 arcmin diamteter of the Coma cluster. This sample contains $36 \mathrm{dEs} / \mathrm{dS} 0 \mathrm{~s}$. We also include velocity dispersion measurements from the literature (see Figure 1). The $L-\sigma$ relation exhibits a change of slope where $L \propto \sigma^{2.01 \pm 0.36}$ for faint early-type galaxies, while the bright early-type galaxies seem to follow the FJ relation.

One of the possible reasons for the change of slope in the $L-\sigma$ relation may be the presence of galaxies other than elliptical. We used the bulge-to-total $(\mathrm{B} / \mathrm{T})$ ratios from Gutiérrez et al. (2004) to classify the galaxies in this combined sample as bulgedominated for $B / T=1$, bulge+exponential component for $0.5<B / T<1.0$, and single exponential component galaxies for $B / T<0.5$. When we fit a $L-\sigma$ relation for each of these classes of galaxies, we observe a slight difference in slope. However, this difference is not sufficient to explain the change of slope in the $L-\sigma$ relation from bright to faint early-type galaxies. Furthermore, it is difficult to classify galaxies at the distance of the Coma cluster without resolved photometry.

Next, we investigate the possibility of rotation as the cause of the change of slope in the $L-\sigma$ relation. Assuming a universal relation between L, kinetic energy per unit mass, $V^{2}$, one can derive a relation between $\mathrm{L}, \sigma$, and the anisotropy parameter, $V_{\text {rot }} / \sigma$. For bright elliptical galaxies $L=b \sigma^{4}$ implies $V_{\text {rot }}=0$, as is observed. However, for the faint early-type galaxies, $L=c \sigma^{2}$ implies a systematic increase in the anisotropy parameter:

$$
\frac{V_{r o t}^{2}}{\sigma^{2}}=\sqrt{\frac{c}{b}} \frac{1}{\sigma}-3
$$

$\dagger$ Throughout this paper we use $H_{0}=70 \mathrm{~km} \mathrm{~s}^{-1} \mathrm{Mpc}^{-1}$, and a distance modulus for Coma cluster of $35.078,(d=99 \mathrm{Mpc})$. 


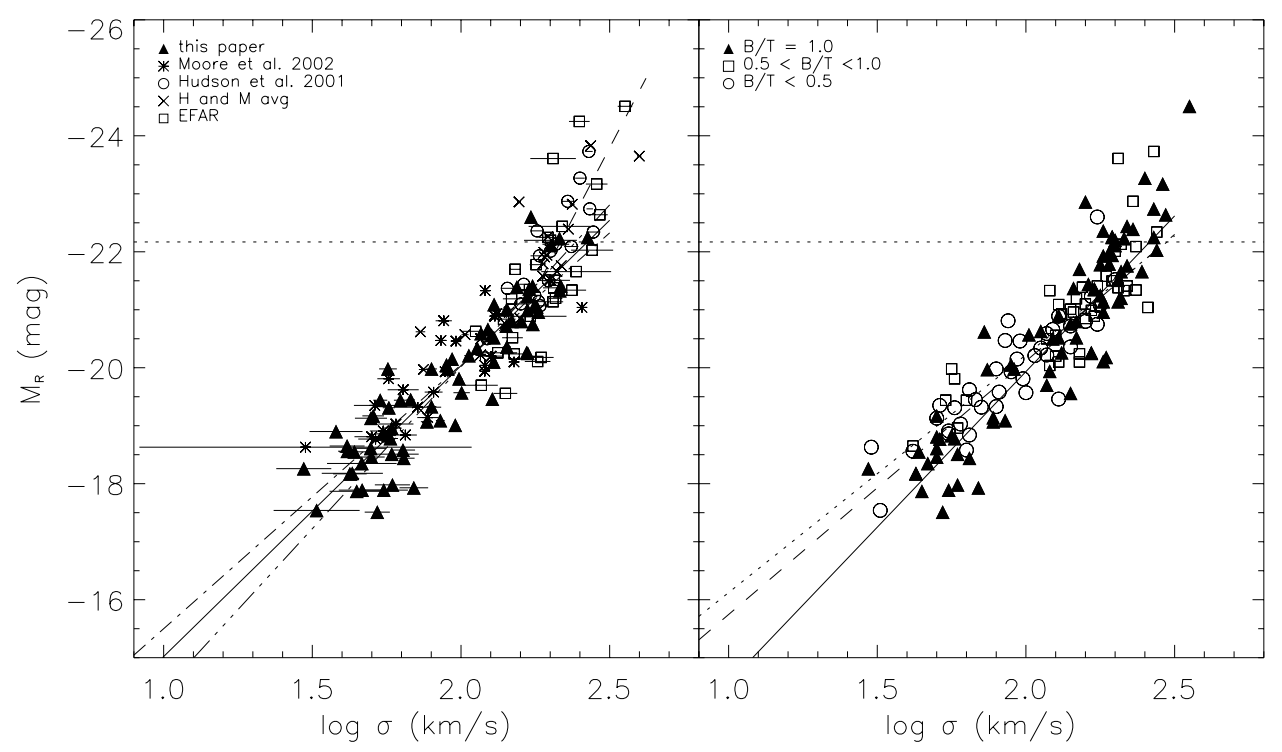

Figure 1. $M_{R}-\sigma$ plots. (Left) Coma galaxies in our sample and from the literature, as indicated in the legend. The dotted line separates the bright from faint early-type galaxies at $M_{R}=-22.17$ mag, while the dashed line is the most recent Faber-Jackson relation from the literature (Forbes \& Ponman, 1999). The solid line is the least squares bisector fit to all faint early-type galaxies. (Right) Coma cluster galaxies for which bulge-to-total (B/T) ratios were available (Gutiérrez et al. 2004). The solid line is the bisector fit to the single exponential component $(B / T<0.5)$ galaxies, the dashed line for bulge+single component $(0.5<B / T<1.0)$, and the dotted line bulge-dominated $(B / T=1)$ galaxies.

In Figure 2 the solid line shows this relation. It represents the change in the anisotropy parameter with velocity dispersion, assuming that rotation was solely responsible for the change of slope in $L-\sigma$. It is crucial to note that previously observed galaxies (Geha, Guhathakurta \& van der Marel 2003; Simien \& Prugniel 2002; Pedraz et al. 2002; Davies et al. 1983) do not follow this relation. Therefore, rotation cannot explain the change of slope in $L-\sigma$ for these faint early-type galaxies.

De Rijcke et al. (2004) also find that $L \propto \sigma^{2}$ for their sample of dEs and show that this is consistent with galactic wind models.

\section{Ages and Metallicities}

We also measured line strength indices for our sample of faint early-type galaxies. Preliminary investigation of relations between different combinations of age and metallicity sensitive indices with the velocity dispersion hint that the faintest galaxies, dEs/dS0s, may exhibit a wider range in metallicity and age than the remaining early-type galaxies.

In Figure 3, we plot the age sensitive index, $\mathrm{H} \beta$, vs. the metallicity sensitive index, $[\mathrm{MgFe}]$. We represent dEs/dS0s as triangles, while asterisks are other faint early-type galaxies. Overall, the galaxies show a wide range in age and metallicity. The dwarf galaxies span a wider range in both age and metallicity with some lying outside the stellar synthesis model grids. These are potentially very old, low metallicity dwarf galaxies whose stellar populations may be similar to those of globular clusters. 


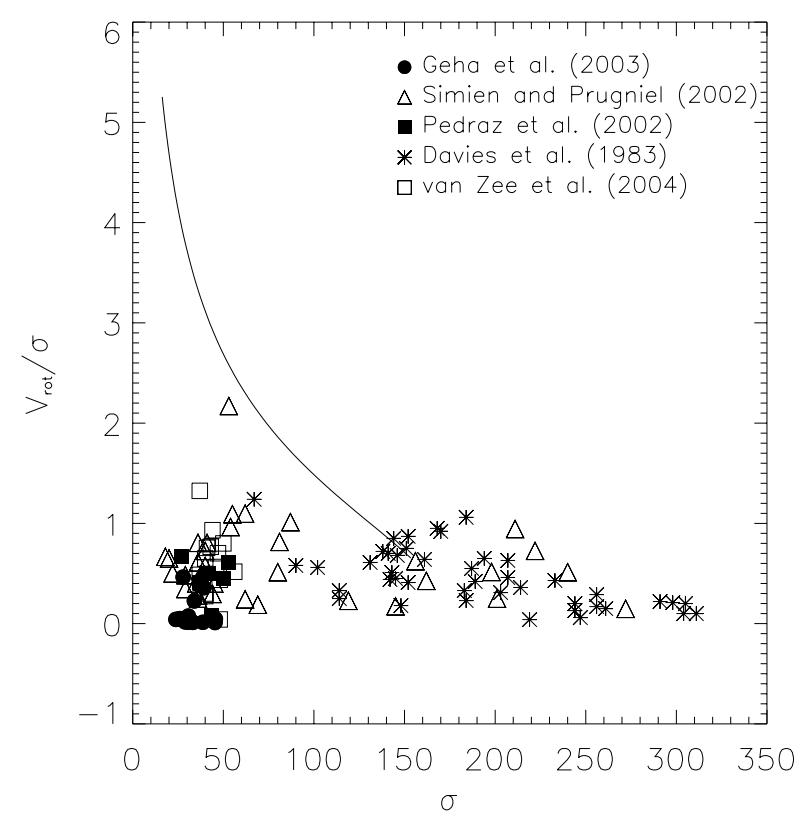

Figure 2. $V_{\text {rot }} / \sigma$ vs. $\sigma$ plot. The solid line represents the expected systematic increase in the anisotropy parameter assuming that rotation is responsible for the change of slope in the $L-\sigma$ relation. The different symbols are observations from the literature as indicated in the legend.

\section{Summary}

- We show preliminary results of a larger environmental study intended to characterize internal kinematics and stellar populations of faint early-type galaxies in the Coma cluster.

- We find that $L \propto \sigma^{2.01 \pm 0.36}$ for faint early-type galaxies $\left(-15.8>M_{B}>-20.5\right)$, while the bright elliptical galaxies follow the FJ relation well. After investigating possible causes of this change of slope, we conclude that rotation cannot explain it.

- The $\mathrm{H} \beta$ vs. $[\mathrm{MgFe}]$ plot indicates that all the faint early-type galaxies in our sample exhibit a wide range in metallicity and age, with dEs/dS0s showing a wider range in these properties. A few galaxies in this sample fall outside the stellar synthesis grids and may be old, low metallicity systems reminiscent of stellar populations of globular clusters.

\section{Acknowledgements}

We would like to acknowledge Patricia Sánchez-Blázquez, Javier Gorgas, and Nicolás Cardiel for help with the velocity dispersion and line strength index measurements. We also thank Alister Graham for many insightful discussions.

\section{References}

Cardiel, N. 1999, Dissertation, Star formation in Central Cluster Galaxies, Univ. Complutense, Madrid

De Rijcke, S., Michielsen, D., Dejonghe, H., Zeilinger, W.W. \& Hau, G.K.T. 2004, astroph/0412553, v2

Davies, R.L., Efstathiou, G., Fall, M., Illingworth, G. \& Schechter, R.L. 1983, AJ 266, 41

Faber, S.M. \& Jackson, R.E. 1976, ApJ 204, 668 


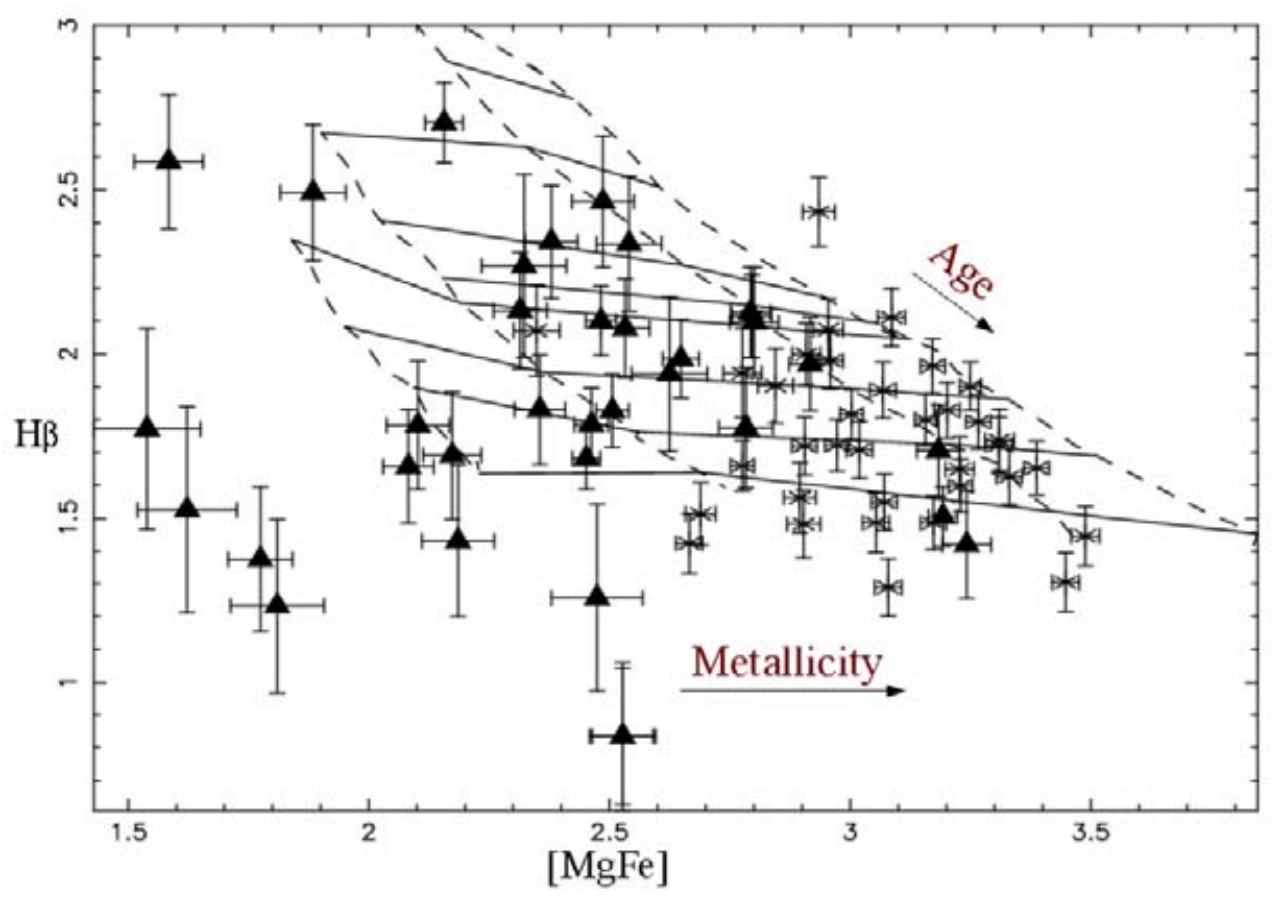

Figure 3. $\mathrm{H} \beta$ vs. $[\mathrm{MgFe}]$ plot. The solid triangles are galaxies classified as dwarf galaxies $\left(M_{B}>-18\right)$, while asterisks are the remaining galaxies in our sample. The ages represented by solid lines, top-to-bottom, are: $1.78,2.51,3.16,3.98,5.62,7.98,12.2,15.85 \mathrm{Gyr}$; while the metallicity lines are dashed, left-to-right, $[\mathrm{Fe} / \mathrm{H}]=-0.68,-0.38,0.0,+0.2$ dex.

Ferguson, H.C. \& Binggeli, B. 1994, A\&A Rev. 6, 67

Forbes, D.A. \& Ponman, T.J. 1999, MNRAS 309, 623

Geha, M., Guhathakurta, P. \& van der Marel, R. 2003, AJ 126, 1794

Gutiérrez, C.M., Trujillo, I., Aguerri, J.A., Graham, A.W. \& Caon, N. 2004, ApJ 602, 644

Held, E.V., de Zeeuw, T., Mould, J. \& Picard, A., 1992, AJ 103,851

Matković, A. \& Guzmán, R. 2005, MNRAS submitted

Pedraz, S., Gorgas, J., Cariel, N., Sanchez-Blazquez, P. \& Guzmán, R. 2002, MNRAS 332, L59

Sargent, W.L.W., Schechter, P.L., Boksenberg, A. \& Shortridge, K. 1977, ApJ 212, 326

Schechter, P.L. \& Gunn, J. E. 1979, ApJ 229, 472

Schechter, P.L. 1980, AJ 85, 801

Simien, F. \& Prugniel, P. 2002, A\& A 384, 371

Smith, R.J. et al. 2004, AJ 128, 1558

Terlevich, A.I., Davies, R.L., Faber, S.M. \& Burstein, D. 1981, MNRAS 196, 381

Tonry, J.L. 1981, ApJ 251, 1

Tonry, J.L. \& Davis, M. 1981, ApJ 246, 680

van Zee, L., Skillman, E.D. \& Haynes, M.P. 2004, AJ 128, 121

\section{Discussion}

N. CALDWELl: Your result that the lower velocity dispersion galaxies have a large spread in age is also found for Virgo and field galaxies, using similar techniques (Caldwell et al. 2003). Also, the galaxies which fall off the grid would appear to lie in the area occupied 
by globular clusters. Though this is a shortcoming of the models, it may mean that those galaxies have populations similar to those of globular clusters.

Matkovic: Yes, I completely agree with your findings in Virgo, and I dosee the possibility of some of these dwarf galaxies having stellar populations similar to those of globular clusters as I mentioned in the talk.

C. Conselice: At what magnitude to you begin to see a spread in ages and metallicities? Is it a range at all magnitudes?

MATKOviC: The spread in both age and metallicity is seen over all the magnitudes. Keep in mind that our sample does not include galaxies fainter than $M_{B} \sim-15.8$.

HELD: Your results on the $L-\sigma$ relation for dwarf elliptical galaxies interestingly confirm - as does De Rijcke's talk - the result obtained by Held et al. (1992, AJ) based on the much smaller dataset available at that time.

MatKovic: Thank you very much for pointing out this reference.

SiLVA: Did you correct for $\mathrm{H} \beta$ emission?

Matkovic: Yes, that is important, however in the Coma cluster we observed, we do not see any emission.

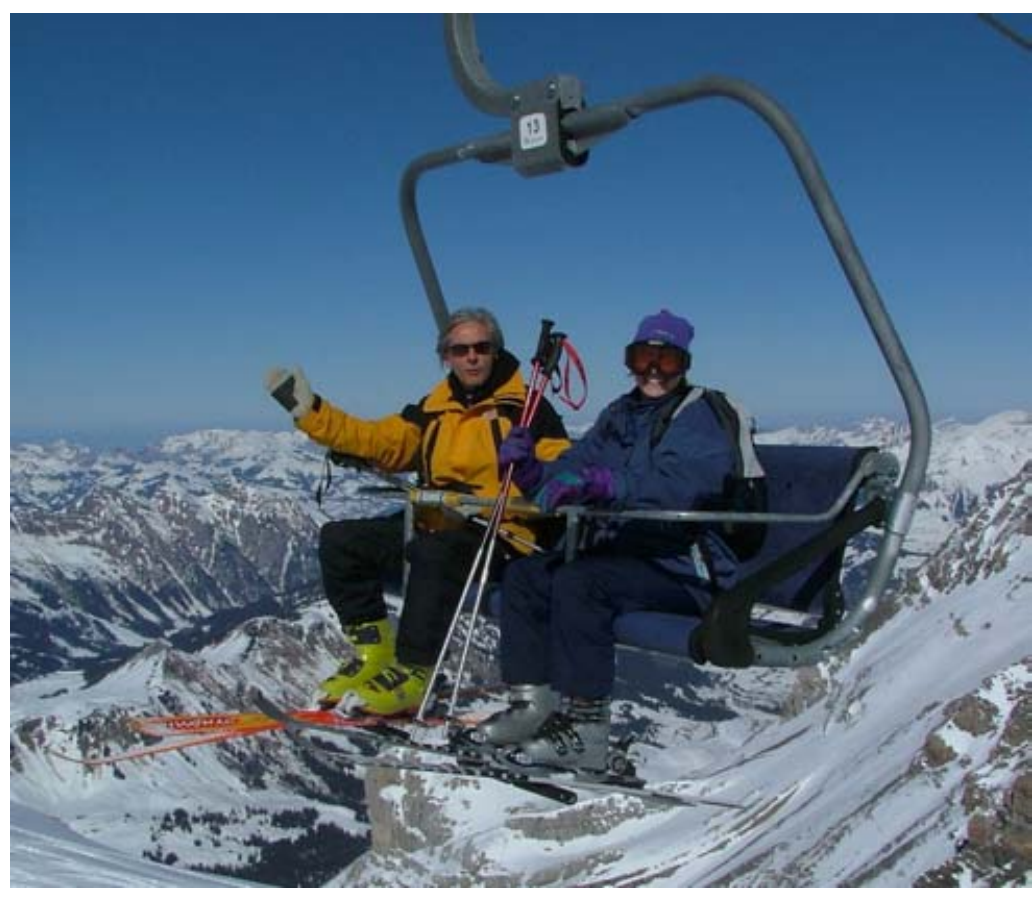

